\title{
Clinical Study \\ Species Distribution and Susceptibility to Azoles of Vaginal Yeasts Isolated Prostitutes
}

\author{
Norma T. Gross, ${ }^{1}$ M. L. Arias, ${ }^{1}$ M. Moraga, ${ }^{2}$ Y. Baddasarow, ${ }^{2}$ and C. Jarstrand ${ }^{3}$ \\ ${ }^{1}$ Centro de Investigaciones en Enfermedades Tropicales (CIET), Facultad de Microbiologia, Universidad de Costa Rica, \\ San José 2060, Costa Rica \\ ${ }^{2}$ Departamento de Control de SIDA y Enfermedades de Transmisión Sexual, Caja Costarricense de Seguro Social, \\ San José 1000, Costa Rica \\ ${ }^{3}$ Karolinska Institutet, Department of Laboratory Medicine F72, Karolinska University Hospital, 14186 Stockholm, Sweden
}

Received 23 April 2007; Accepted 29 May 2007

Objective. We investigated the use of miconazole among female prostitutes in Costa Rica as well as the distribution of vaginal yeasts and the susceptibility pattern to azoles of strains obtained from this population. Our intention was to relate a frequent use of miconazole to occurrence of vaginal yeasts resistant to azoles. Methods. Vaginal samples were taken from 277 patients that have previously used azoles. Vaginal swabs were obtained for direct microscopy and culture. Yeast isolates were identified by germ tube test and assimilation pattern. Susceptibility testing was determined using a tablet diffusion method. Results. The number of clinical Candida isolates (one from each patient) was 57 (20.6\%). C. albicans was the predominant species (70\%), followed by C. parapsilosis (12\%), C. tropicalis (5.3\%), C. glabrata and C. famata (3.5\% each), C. krusei, C. inconspicua and C. guilliermondii (1.7\% each). The majority of vaginal Candida isolates were susceptible to ketoconazole (91\%), fluconazole (96.5\%), and itraconazole (98\%). A lower susceptibility of some isolates to miconazole (63\%) was observed as compared to the other azoles tested. Moreover, the strains, nonsusceptible to miconazole, were more often obtained from patients that have used this antifungal at least four times within the last year before taking the samples as compared to those with three or less treatments $(P<.01)$. Conclusion. An indiscriminate use of miconazole, such as that observed among female prostitutes in Costa Rica, results in a reduced susceptibility of vaginal yeasts to miconazole but not to other azoles.

Copyright (c) 2007 Norma T. Gross et al. This is an open access article distributed under the Creative Commons Attribution License, which permits unrestricted use, distribution, and reproduction in any medium, provided the original work is properly cited.

\section{INTRODUCTION}

Candida may be either a commensal or a pathogen of the vagina, a fact which indicates that changes in the vaginal microenvironment are generally necessary for Candida to induce pathological changes associated with clinical symptoms [1]. Vulvovaginal candidosis (VVC) is a common cause of vaginal discharge, soreness, vulval burning, dysuria and local pruritus in women. C. albicans is the most common etiological agent of VVC, but other species such as C. glabrata, $C$. krusei, and C. tropicalis are also encountered [1-3].

Depending on the age, locality, and social economical status, the frequency of vaginal yeast isolates has been reported to be between $5 \%$ and $48.4 \%$ in healthy nonpregnant women [4-6]. During pregnancy, an increase of $7.8 \%$ and $67.8 \%$ of yeast vaginal isolates has been reported $[7,8]$. A study in Jordan revealed a different distribution of strains of Candida spp. among pregnant, infertile, and (nonpregnant, fertile) healthy controls [6].
Azoles are antifungal drugs frequently used for treatment of VVC. There is evidence, however, of an increased azole resistance among isolates of Candida spp. isolated from patients with VVC $[9,10]$. Non-C. albicans are generally more resistant to azoles than are C. albicans strains [11]. Particularly in patients with VVC, several authors have found an increased prevalence of C. glabrata due to a selection mechanism related to the frequent use of imidazoles (ketoconazole and miconazole) $[5,12,13]$. VVC caused by a C. albicans strain, resistant to fluconazole after azole treatment, has been reported [14]. Several authors using genetic analysis have shown that the most common factor seen in recurrent VVC is the development of resistance during treatment $[15,16]$.

In the present work, we wanted to study the occurrence of vaginal yeasts, their species distribution, and the susceptibility pattern to azoles of the strains obtained from female prostitutes who attended at a public clinic in San José, Costa Rica. Since an indiscriminate use of self-prescribed azoles is 
frequent in this population, our aim of this study was also to determine if this behavior might cause resistance of infecting yeasts to these antifungal drugs.

\section{SUBJECTS AND METHODS}

\subsection{Patients}

Vaginal samples were collected during 24 months from 277 prostitutes examined at the Control Center of AIDS and Sexual Transmitted Diseases, Caja Costarricence del Seguro Social, San José, Costa Rica. The study included patients with or without clinical symptoms of vulvovaginitis (pruritus, dysuria, or vulval burning). All patients had previously taken miconazole ( $\geq 1-\leq 3$ and $\geq 4-\leq 10$ times) within the last year before the clinical samples were taken. Each patient completed a questionnaire about age, history of diabetes, and use of contraceptives, vaginal douche, and antimicrobial agents. Vulvovaginal symptoms and findings at the gynecological examination such as type of secretion, vulval oedema, and vulval erythema were recorded.

\subsection{Sampling procedure}

Two sterile swabs were obtained from each patient. One of the swabs was inoculated into Sabouraud dextrose agar containing penicillin $\left(0.01 \mathrm{~g} \mathrm{~mL}^{-1}\right)$ and streptomycin $\left(0.03 \mathrm{~g} \mathrm{~mL}^{-1}\right)$. The plates were then incubated at room temperature $\left(25-30^{\circ} \mathrm{C}\right)$ for 5 days. The second swab was first streaked into a glass slide for Gram preparation, and then placed in a tube containing $0.5 \mathrm{~mL}$ of sterile $0.85 \%$ saline solution. A wet saline mount and a $20 \%$ potassium hydroxide preparation of the sample were then mounted on glass slides for the observation of trichomonas and yeast cells and/or hyphae in a light microscope using low magnification.

\subsection{Identification of the yeast isolates}

Yeast cultures were identified as C. albicans by development of germ tubes in human serum. Germ tube-negative isolates were identified by their assimilation pattern with the ID 32C identification system for yeasts (BioMerieux, France). The identified yeast isolates were maintained in distilled water at $5^{\circ} \mathrm{C}$.

\subsection{Susceptibility testing}

The tablet diffusion method (Neo-Sensitabs, ROSCO Diagnostica, Taastrup, Denmark $[17,18]$ ) was performed for all isolates according to the manufacturer's instructions. An inoculum containing approximately $5 \times 10^{5}$ colony forming units (CFU) $\mathrm{mL}^{-1}$ (McFarland 0.5, diluted 1:1 with $0.85 \%$ saline solution or diluted 1:10 in the case of C. krusei) was prepared. One $\mathrm{mL}$ of this suspension was poured onto the agar surface (flooding) and excess liquid was removed immediately with a pipette. Thereafter, the open plates were dried at $37^{\circ} \mathrm{C}$ for 15 minutes and the tablets were placed on the agar surface. The azoles tested were miconazole, ketoconazole, fluconazole, and itraconazole. Plates were incubated at $37^{\circ} \mathrm{C}$ for 18 hours and the inhibition zones were measured. C. albicans ATCC 64548 and ATCC 64550 were included as the quality controls for susceptibility testing. Isolates were classified as susceptible, intermediate, and resistant according to the manufacturer's recommendations as follows: for miconazole, ketoconazole, and fluconazole, susceptible $=\geq 20 \mathrm{~mm}$, intermediate $=12-19 \mathrm{~mm}$, resistant $=\leq 11 \mathrm{~mm}$ and for itraconazole, susceptible $=\geq 15 \mathrm{~mm}$, intermediate $=10-14 \mathrm{~mm}$, resistant $=$ no zone.

Yeast isolates classified by the tablet diffusion method as intermediate or resistant to fluconazole were tested according to the microdilution method proposed by the European Committee on Antibiotic Susceptibility (EUCAST) [19]. Fluconazole powder was provided by Pfizer Inc. (Stockholm, Sweden). Results for miconazole were only obtained by the tablet diffusion method since susceptibility testing to this antifungal has not yet been standardized by either the M-27 A2 reference method of NCCLS (now CLSI, Clinical Laboratory Standard Institute) [20] or the EUCAST.

\section{RESULTS}

\subsection{Patients}

Of the 277 patients examined, 106 were symptomatic (38\%) and 171 were asymptomatic (62\%). Among patients with symptoms, $48 \%$ had only one of the symptoms (itching, vulvar burning, or dysuria), $32 \%$ and $20 \%$ had two and three symptoms, respectively (Table 1). The age range of the patients was from 18 to 50 years and the mean age was 30 years. Only 12 patients $(4.3 \%)$ were taking antibacterial treatment. Two patients $(0.72 \%)$ were diabetic. Vaginal douche was a common practice in $122(44 \%)$ of the patients. For fertility protection, some of the patients used more than one method with 105 patients taking estrogens, 16 using intrauterine devices, 118 using condoms, 32 were protected by salpingectomy and 70 unprotected.

\subsection{Identification of the yeast isolates}

The number of patients with clinical Candida isolates (each patient was counted only once) was $57(20.6 \%)$. Of these isolates 36 were found among patients with symptoms and 21 from those who were asymptomatic (Table 1). C. albicans was the predominant species (70\%), followed by C. parapsilosis $(12 \%)$. The identity of all Candida species isolated is given in detail in Table 2. It was found that only 36 (34\%) out of 106 vaginal samples obtained from patients with vulvovaginitis were culture positive for Candida spp. Thirty-one patients with symptoms had negative yeast cultures and reported the use of condom. Twenty-nine patients with symptoms had negative yeast cultures and reported the use of acetic acid in vaginal douche. Trichomonas vaginalis and Gram-negative pleomorphic bacilli were reported in 6 and 2 patients with negative yeast cultures, respectively. 
TABLE 1: Candida isolates in patients without $(n=171)$ or with different symptoms of vulvovaginitis $(n=106)$ all with previous miconazole treatment.

\begin{tabular}{lccc}
\hline Symptoms & Number of patients & Number of Candida isolates & Number of miconazole nonsusceptible strains \\
\hline Itching & 45 & - & - \\
Vulval burning or dysuria & 6 & - & - \\
Itching, vulval burning, and dyuria & 21 & 36 & - \\
Summary of patients with symptoms & 106 & 21 & 6 \\
Patients without symptoms & 171 & 13 \\
\hline
\end{tabular}

TABLE 2: Azole nonsusceptibility of Candida isolates from vagina of women with or without vulvovaginitis and with previous miconazole treatment. Total number of isolates $=57$.

\begin{tabular}{|c|c|c|c|c|c|}
\hline Yeast & $\begin{array}{l}\text { Number of } \\
\text { isolates }\end{array}$ & $\begin{array}{c}\text { Fluconazole } \\
\text { nonsusceptible }\end{array}$ & $\begin{array}{c}\text { Itraconazole } \\
\text { nonsusceptible }\end{array}$ & $\begin{array}{c}\text { Ketoconazole } \\
\text { nonsusceptible }\end{array}$ & $\begin{array}{c}\text { Miconazole } \\
\text { nonsusceptible }\end{array}$ \\
\hline C. albicans & 40 & - & - & - & 10 \\
\hline C. parapsilosis & 7 & - & - & - & 5 \\
\hline C. tropicalis & 3 & - & - & - & 2 \\
\hline C. glabrata & 2 & 1 & 1 & 2 & 2 \\
\hline C. famata & 2 & - & - & - & - \\
\hline C. krusei & 1 & 1 & - & 1 & 1 \\
\hline C. inconspicua & 1 & - & - & - & 1 \\
\hline C. guillermondii & 1 & - & - & - & - \\
\hline Total number & 57 & 2 & 1 & 3 & 21 \\
\hline
\end{tabular}

\subsection{Susceptibility testing}

Among the different species of Candida, resistance to fluconazole (MIC, $>64 \mu \mathrm{g} / \mathrm{mL}$, EUCAST method) was observed with one isolate of C. krusei and another one of C. glabrata. The C. krusei isolate had zone diameters for fluconazole, ketoconazole, and miconazole $\leq 11 \mathrm{~mm}$ (resistant), and $22 \mathrm{~mm}$ for itraconazole (susceptible). The C. glabrata isolate had zone diameters for fluconazole, ketoconazole, and miconazole $\leq 11 \mathrm{~mm}$ and no zone for itraconazole (resistant to all azoles tested). Three isolates intermediate to ketoconazole were observed. The isolated strains were C. albicans (2) and C. glabrata (1). As to miconazole, resistant isolates were identified as C. parapsilosis (2), C. albicans (1), and C. glabrata (1). The strains intermediate to miconazole were C. albicans (9), C. parapsilosis (3), C. tropicalis (2), C. glabrata (1), and C. inconspicua (1).

Tables 2 and 3 summarize the in vitro susceptibility of 57 Candida strains to fluconazole, itraconazole, ketoconazole, and miconazole as determined by a tablet diffusion test. Table 1 shows that miconazole resistant strains were found to a high degree among asymptomatic as well as among patients with symptoms. In Table 3 , the isolates were divided into two groups, those from women treated with miconazole $\leq 3$ and those treated $\geq 4$ times during the last year before taking the samples. When the two groups containing $22(\leq 3)$ and 35 isolates $(\geq 4)$ were compared, $3(13.6 \%)$ and 18 isolates $(51.4 \%)$, respectively, were nonsusceptible to miconazole. The difference is significant $(P<.01$ Fisher's exact test [21]).

\section{DISCUSSION}

In Costa Rica, the use of miconazole is very frequent among prostitutes as a treatment for vaginal candidasis. It is also a common belief among this group of women that the constant use of antifungal will prevent yeast vaginal infection. The present study was therefore undertaken to examine the occurrence and species distribution of vaginal yeasts and to evaluate susceptibility of yeast isolates recovered from vaginal samples to miconazole. Susceptibility testing to ketoconazole, fluconazole, and itraconazole was also performed. In contrast to other azoles, miconazole possesses two mechanisms of action and yeasts are most often found to remain susceptible even after repeated exposures [22]. Our study shows however that after more than three treatment periods miconazole resistant strains are frequently found.

Our particular patient population always chose miconazole and used it even without clinical symptoms of vulvovaginitis. The misuse of this drug is reflected by the fact that resistant Candida strains were found to a high degree among asymptomatic patients (Table 1). The reasons for the choice of miconazole are that this drug, in Costa Rica, is available over-the-counter and that it is less expensive than other azoles. A control group, that is patients that had taken repeated treatments (more than three times) of azoles other than miconazole, was not possible to obtain. However, our patients with three treatments or less can be considered as controls to those with four or more treatments. A significantly higher number of nonsusceptible yeast isolates were found in this latter group than among the controls (Table 3 ). 
TABLE 3: Azoles susceptibility pattern of Candida spp. isolated from vagina of women with or without vulvovaginitis with previous miconazole treatment ( $\leq 3$ or $\geq 4$ times). Total number of isolates $=57$ (consisting of 22 and 35 isolates from women taken miconazole $\leq 3$ and $\geq 4$ times, respectively, during the last year before taking the samples). When the two groups ( $\leq 3$ and $\geq 4$ ) were compared as to miconazole nonsusceptibility, the $P$ value was $<.01$.

\begin{tabular}{lcccccccc}
\hline & \multicolumn{2}{c}{ Resistant } & \multicolumn{2}{c}{ Intermediate } & Nonsusceptible (resistant + intermediate) & \multicolumn{2}{c}{ Susceptible } \\
& $\leq 3$ & $\geq 4$ & $\leq 3$ & $\geq 4$ & $\leq 3$ & 1 & $\leq 3$ \\
\hline Fluconazole & 1 & 1 & 0 & 0 & 1 & 0 & 20 \\
Itraconazole & 1 & 0 & 0 & 0 & 1 & 2 & 35 \\
Ketoconazole & 1 & 1 & 2 & 1 & 3 & 18 & 36 \\
Miconazole & 1 & 4 & 2 & 14 & 3 & 34 & 18 \\
\hline
\end{tabular}

A previous study in female sexual workers revealed that the most frequently isolated vaginal yeast was $C$. albicans, followed by C. glabrata [10]. In the general population, several studies also indicate that C. albicans predominates over other species. C. glabrata is the second one most common, but other species such as C. krusei, C. parapsilosis, C. tropicalis, C. guilliermondii, C. kefyr are also encountered $[2,3,6]$. In accordance with these previous authors, in our study $C$. albicans was the dominant vaginal isolate. However, C. parapsilosis was the second one most common, followed by $C$. tropicalis and then by C. glabrata.

Clinical symptoms such as itching, vulvar burning, and dysuria are not always indicative of VVC [1], which is in agreement with our results. Among possible explanations for these findings are contact dermatitis or allergic reactions probably related to the use of condom or other devices such as acetic acid used in vaginal douche. Other causative agents could be involved, such as Trichomonas vaginalis or Gramnegative pleomorphic bacilli. These observations are important to consider for adequate treatment of vulvovaginitis.

In a previous in vitro susceptibility study of vaginal isolates in Jordan, miconazole nitrate was less effective as compared to amphotericin $\mathrm{B}$, nystatin, and chlorhexidine. In the study, however, no other azoles were examined [6]. The susceptibility to fluconazole of C. albicans and C. glabrata isolated from female prostitutes with vulvovaginitis was evaluated in another study [10]. These authors reported a greater susceptibility to fluconazole for C. albicans compared to C. glabrata. They isolated one (4.3\%) resistant strain of C. glabrata and four dose-dependent susceptible strains (17.3\%).

The reference method for susceptibility testing to azoles (fluconazole, ketoconazole, and itraconazole) is the document M27-A2, proposed by CLSI [20]. However, susceptibility testing to miconazole has not been standardized. The method used in the present study, the tablet diffusion (Neosensitabs), is an alternative method which offers susceptibility testing to miconazole with interpretation of the zone diameters for local infections. Thus, the results of our study were analyzed in accordance with this method.

Concerning the susceptibility of the vaginal isolates to azoles, our data indicate that the majority of the isolates were susceptible to the azoles evaluated. Resistance to fluconazole was observed with one isolate of C. krusei. The isolate was also resistant to ketoconazole and miconazole, but not to itraconazole. It is well-established that C. krusei is intrinsically resistant to fluconazole and ketoconazole [23, 24]. As to itraconazole, C. krusei is usually susceptible [21]. One isolate of C. glabrata also exhibited resistance to fluconazole and the rest of the azoles tested. It is known that $C$. glabrata can develop resistance to fluconazole as a primary and secondary mechanism [24].

In the present study, miconazole which was the azole used by all patients showed the lowest percentage of susceptibility $(63 \%)$. It is also of interest that $9 \%$ of the isolates were resistant and $28 \%$ were intermediate to miconazole. Further, most of the nonsusceptible strains to miconazole were obtained from women that had been taking this antifungal four or more times during the last year before taking the clinical samples. In our study, there was only one C. albicans resistant isolate. However, among the isolates classified as intermediate, there were 9 C. albicans. Our finding is in agreement with previous observations that the majority of yeast isolates that develop resistance while on treatment are C. albicans [25-28]. Taking these observations into account, it is recommended to advice this group of women not to take antimicrobials indiscriminatively.

In conclusion, the great majority of vaginal Candida isolates exhibited susceptibility to ketoconazole, fluconazole, and itraconazole. However, a tendency for lower susceptibility of some isolates to miconazole was observed as compared to the other azoles tested, a finding that could be related to the abuse of miconazole treatment by female prostitutes in Costa Rica.

\section{ACKNOWLEDGMENTS}

The authors are thankful to Liliana Zumbado for helping with the collection of vaginal samples. They are indebted to Mariela Campos and Vanessa Castro for also helping with the collection of clinical samples and for valuable help at the laboratory. The study was supported by Grant no. 803-A1-108 from the University of Costa Rica, Central America.

\section{REFERENCES}

[1] J. D. Sobel, "Vaginitis," New England Journal of Medicine, vol. 337, no. 26, pp. 1896-1903, 1997.

[2] H. L. Kent, "Epidemiology of vaginitis," American Journal of Obstetrics and Gynecology, vol. 165, no. 4, pp. 1168-1176, 1991. 
[3] E. A. Sim and C. Hughes, "Candida species from genital sites: their identification and susceptibility to fluconazole," British Journal of Biomedical Science, vol. 55, no. 4, pp. 264-267, 1998.

[4] M. S. A. Harady and A. Mustafa, "Yeast flora of the human vagina and effects of antifungal agents on its growth in vitro," Korean Journal of Mycology, vol. 21, pp. 140-145, 1993.

[5] F. C. Odds, "Epidemiological shifts in opportunistic and nosocomial Candida infections: mycological aspects," International Journal of Antimicrobial Agents, vol. 6, no. 3, pp. 141-144, 1996.

[6] K. H. Abu-Elteen, A. M. M. Abdul Malek, and N. A. Abdul Wahid, "Prevalence and susceptibility of vaginal yeast isolates in Jordan," Mycoses, vol. 40, no. 5-6, pp. 179-185, 1997.

[7] W. H. Robertson, "Mycology of vulvovaginitis," American Journal of Obstetrics and Gynecology, vol. 158, no. 4, pp. 989991, 1988.

[8] V. Vidotto, L. Guevara Ochoa, L.-M. Ponce, G. M. Tello, G. R. Prada, and M. Bruatto, "Vaginal yeast flora of pregnant women in the Cusco region of Peru," Mycoses, vol. 35, no. 9-10, pp. 229-234, 1992.

[9] R. C. Candido, M. R. T. Toloi, S. A. Franceschini, F. R. Garcia, and E. C. M. Minto, "In vitro activity of antimycotic agents determined by E-test method against vaginal Candida species," Mycopathologia, vol. 144, no. 1, pp. 15-20, 1998.

[10] L. Otero, A. Fleites, F. J. Méndez, V. Palacio, and F. Vázquez, "Susceptibility of Candida species isolated from female prostitutes with vulvovaginitis to antifungal agents and boric acid," European Journal of Clinical Microbiology and Infectious Diseases, vol. 18, no. 1, pp. 59-61, 1999.

[11] G. Morace, S. Manzara, and G. Dettori, "In vitro susceptibility of 119 yeast isolates to fluconazole, 5-fluorocytosine, amphotericin B and ketoconazole," Chemotherapy, vol. 37, no. 1, pp. 23-31, 1991.

[12] F. Meunier, “Candidiasis," European Journal of Clinical Microbiology and Infectious Diseases, vol. 8, no. 5, pp. 438-447, 1989.

[13] A. Spinillo, S. Nicola, L. Colonna, E. Marangoni, C. Cavanna, and G. Michelone, "Frequency and significance of drug resistance in vulvovaginal candidiasis," Gynecologic and Obstetric Investigation, vol. 38, no. 2, pp. 130-133, 1994.

[14] J. D. Sobel and J. A. Vazquez, "Symptomatic vulvovaginitis due to fluconazole-resistant Candida albicans in a female who was not infected with human immunodeficiency virus," Clinical Infectious Diseases, vol. 22, no. 4, pp. 726-727, 1996.

[15] K. Schröppel, M. Rotman, R. Galask, K. Mac, and D. R. Soll, "Evolution and replacement of Candida albicans strains during recurrent vaginitis demonstrated by DNA fingerprinting," Journal of Clinical Microbiology, vol. 32, no. 11, pp. 2646-2654, 1994.

[16] S. R. Lockhart, B. D. Reed, C. L. Pierson, and D. R. Soll, "Most frequent scenario for recurrent Candida vaginitis is strain maintenance with "substrain shuffling": demonstration by sequential DNA fingerprinting with probes $\mathrm{Ca} 3, \mathrm{C} 1$, and CARE2," Journal of Clinical Microbiology, vol. 34, no. 4, pp. 767-777, 1996.

[17] J. B. Casals, "Tablet sensitivity testing on pathogenic fungi," Journal of Clinical Pathology, vol. 32, no. 7, pp. 719-722, 1979.

[18] ROSCO, "Neo-Sensitabs, susceptibility testing," A/S Rosco, pp. 18.0-18.3, Taastrup, Denmark, 1998.

[19] European Committee on Antibiotic Susceptibility Testing, Subcommittee on Antifungal Susceptibility Testing. Method for the determination of minimum inhibitory concentration (MIC) by broth dilution of fermentative yeasts, 1999.
[20] National Committee for Clinical Laboratory Standards, Reference method for broth dilution antifungal susceptibility testing for yeasts, proposed standard M27-A2, 2002.

[21] D. J. Finney, R. Latscha, B. M. Bennett, and P. Hsu, Tables for Testing Significance in a $2 \times 2$ Contingency Table, Cambridge University Press, Cambridge, Mass, USA, 1963.

[22] A. W. Fothergill, "Miconazole: a historical perspective," Expert Review of Anti-Infective Therapy, vol. 4, no. 2, pp. 171-175, 2006.

[23] P. Marichal, J. Gorrens, M.-C. Coene, L. LeJeune, and H. Vanden Bossche, "Origin of differences in susceptibility of Candida krusei to azole antifungal agents," Mycoses, vol. 38, no. 34, pp. 111-117, 1995.

[24] T. C. White, K. A. Marr, and R. A. Bowden, "Clinical, cellular, and molecular factors that contribute to antifungal drug resistance," Clinical Microbiology Reviews, vol. 11, no. 2, pp. 382-402, 1998.

[25] F. C. Odds, "Resistance of yeasts to azole-derivative antifungals," Journal of Antimicrobial Chemotherapy, vol. 31, no. 4, pp. 463-471, 1993.

[26] D. Law, C. B. Moore, H. M. Wardle, L. A. Ganguli, M. G. L. Keaney, and D. W. Denning, "High prevalence of antifungal resistance in Candida spp. from patients with AIDS," Journal of Antimicrobial Chemotherapy, vol. 34, no. 5, pp. 659-668, 1994.

[27] K. A. Marr, T. C. White, J.-A. H. van Burik, and R. A. Bowden, "Development of fluconazole resistance in Candida albicans causing disseminated infection in a patient undergoing marrow transplantation," Clinical Infectious Diseases, vol. 25, no. 4, pp. 908-910, 1997.

[28] T. Mori, M. Matsumura, Y. Kanamaru, et al., "Myelofibrosis complicated by infection due to Candida albicans: emergence of resistance to antifungal agents during therapy," Clinical Infectious Diseases, vol. 25, no. 6, pp. 1470-1471, 1997. 


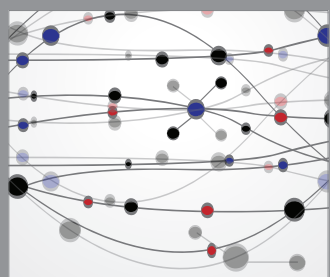

The Scientific World Journal
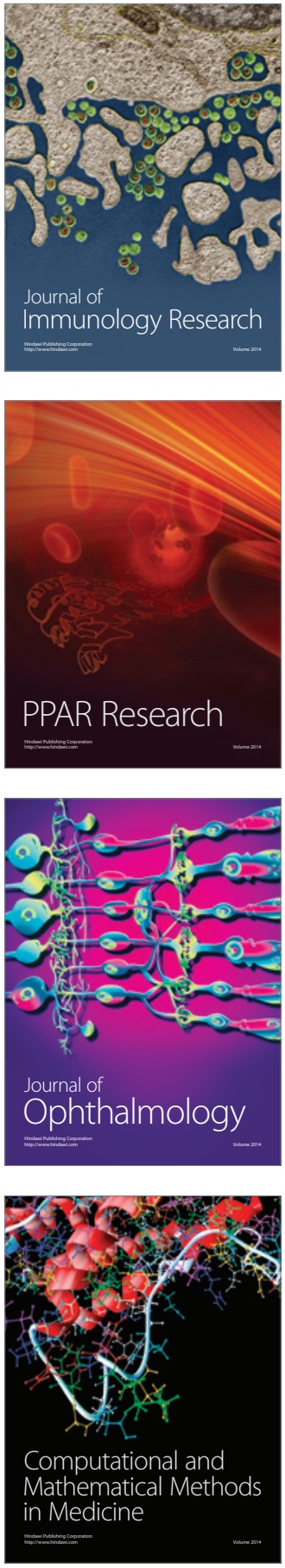

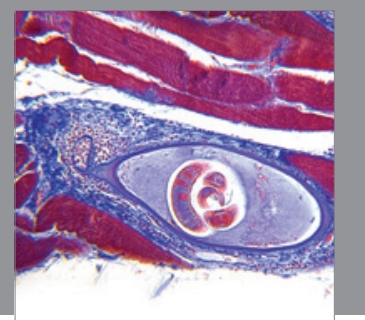

Gastroenterology

Research and Practice
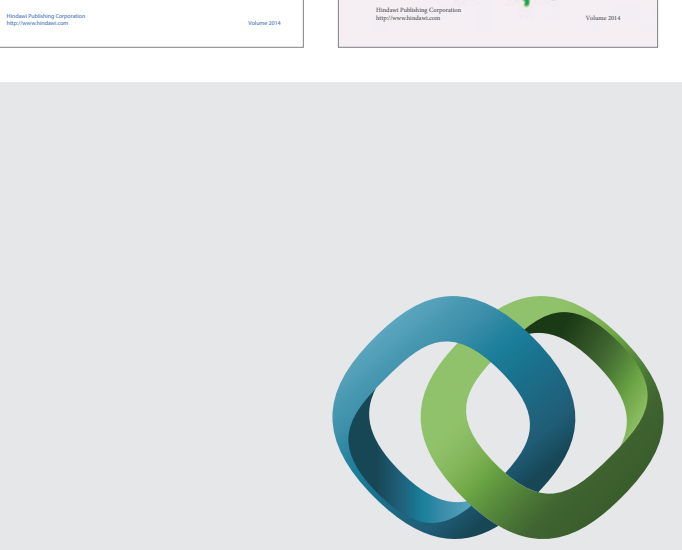

\section{Hindawi}

Submit your manuscripts at

http://www.hindawi.com
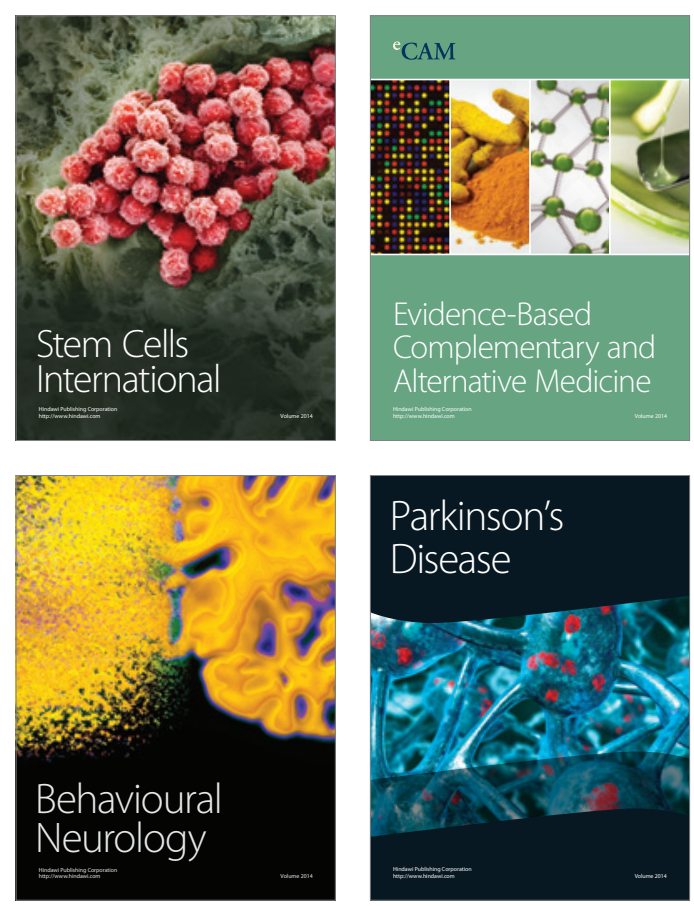

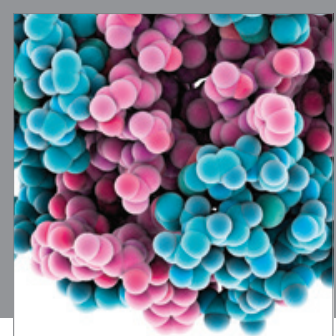

Journal of
Diabetes Research

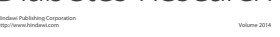

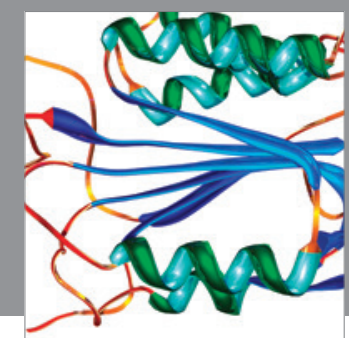

Disease Markers
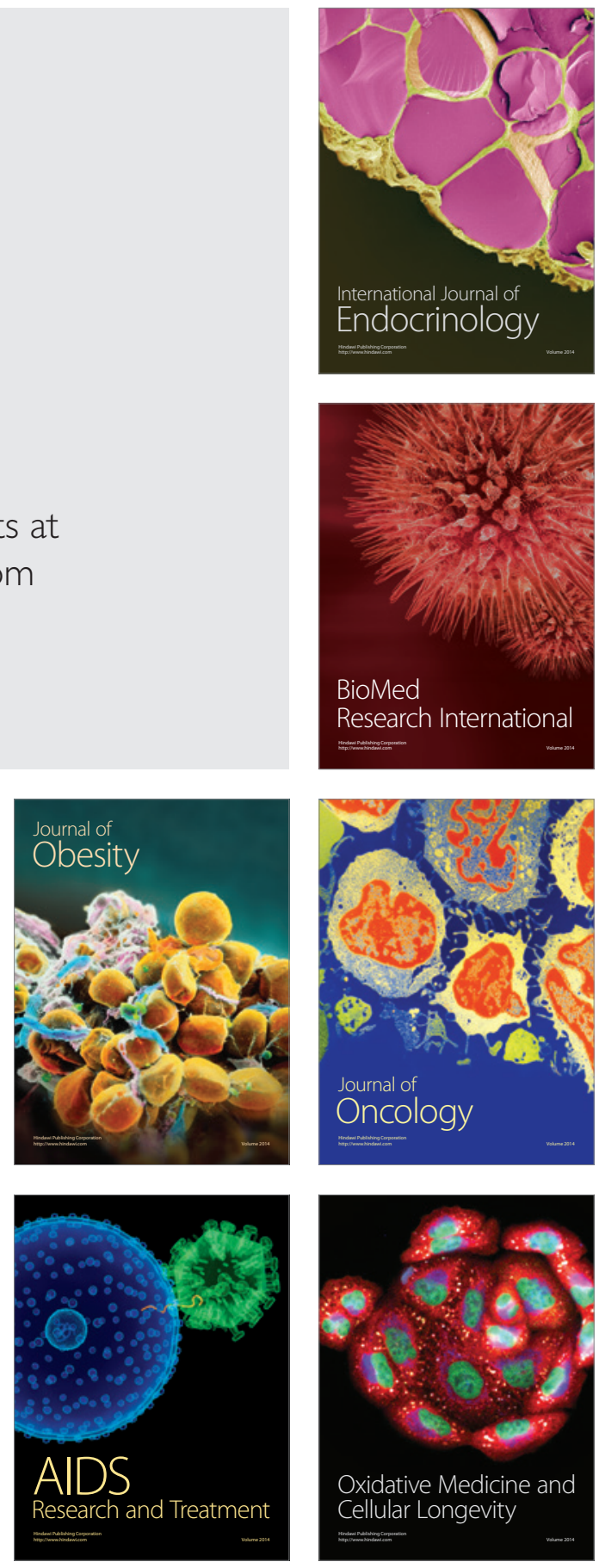